\title{
The Stellar Luminosity Function and Binary Stars
}

\author{
PAVEL KROUPA, CHRISTOPHER A. TOUT, \& GERARD GILMORE \\ Institute of Astronomy, Madingley Road, Cambridge CB3 0HA, England.
}

\begin{abstract}
If all stars within a small volume surrounding the sun are counted we obtain an approximation of the low-mass single-star luminosity function. Alternatively, deep photographic surveys cannot resolve most of the binary systems, and consequently we obtain an approximation to the system luminosity function. Comparing the singlestar and system luminosity functions we derive the stellar mass function and constrain the properties of binary systems.
\end{abstract}

\section{INTRODUCTION}

The faint star luminosity function is interesting for researchers working on dark matter and star formation. Two, in principle, straightforward approaches have been implemented to estimate this function yielding discrepant results. The luminosity function derived by counting the stars within a distance of $5.2 \mathrm{pc}$ has a different shape than the luminosity function determined from photographic surveys at distances of typically $100-200 \mathrm{pc}$. Dahn et al. (1986) raised the possibility that unresolved binary systems in the photographic surveys may be responsible for the difference between the number density of stars in both samples at $M_{\mathrm{V}}>14$. Also, photographic surveys which suffer much less from Poisson uncertainties, lead to luminosity functions with a pronounced peak at $M_{\mathrm{V}} \approx 12$ (see for example Stobie et al. 1989). The nature of this peak remained unknown, but may reflect either structure in the stellar mass function (e.g., Rana 1987), or alternatively may be the result of a minimum in the slope of the mass-absolute-visual-magnitude relation.

\section{THE MASS-LUMINOSITY RELATION}

Small scale structure in the luminosity function can be accounted for by structure in the mass-luminosity relation (Kroupa et al. 1990) as shown in Figure 1. The underlying mass function can be smooth and certainly need not be bimodal. In particular the effect of molecular hydrogen on the equation of state of fully convective stars causes a pronounced peak in the luminosity function at $M_{V} \approx$ +12 and the opacity of $\mathrm{H}^{-}$ions causes the much more subtle dip at $M_{V} \approx+7$.

\section{BINARY STARS}

We find that a large proportion of unresolved binary systems can explain the difference between photographically determined luminosity functions and the nearby luminosity function (Kroupa et al. 1991, Kroupa 1992), as shown in Figure 1 . The following mass function provides a good solution to both luminosity functions:

$$
\xi(m)= \begin{cases}0.035 m^{-\alpha}, & \text { if } 0.08 \leq m \leq 0.5 \\ 0.019 m^{-2.2}, & \text { if } 0.5<m \leq 1.0\end{cases}
$$



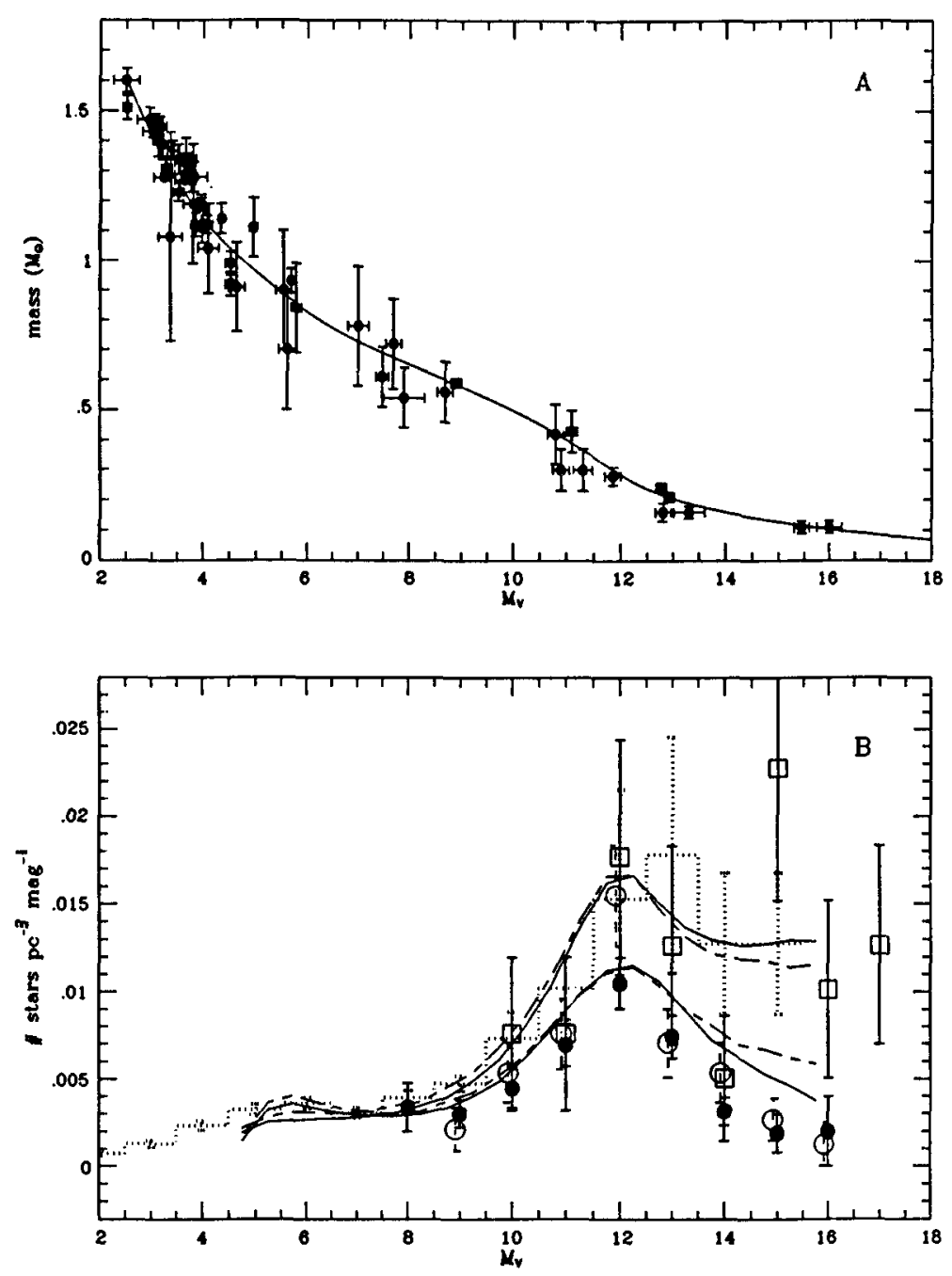

FIGURE 1. Panel A: The mass-luminosity relation (Kroupa 1992). Filled circles are observational data compiled by Popper (1980). Panel B: The luminosity function based on data within 20 pc compiled by Wielen et al. (1983) is shown as a dotted histogram. Open squares represent the determination based on data within $5.2 \mathrm{pc}$ (Kroupa 1992 and references therein). Two examples of photographically determined luminosity functions towards the south galactic pole (Reid \& Gilmore 1982) and the north galactic pole (Stobie et al. 1989) are shown as open and solid circles, respectively. The upper curves depict our single-star Galactic-field models and the lower curves the system luminosity functions for $f=0.2$ (short-long dashed line) and $f=1.0$ (solid line), where $f$ is the proportion of binary systems with two hydrogen burning components the masses of which are chosen independently from the mass function, and $1-f$ is the fraction of systems composed of one main-sequence star (and possibly a dark component). The models are maximum likelihood solutions for $\alpha$ and the disc-scale height $h$. We obtain $\alpha=1.1, h=280 \mathrm{pc}$ for $f=0.2$ and $\alpha=1.3, h=270 \mathrm{pc}$ for $f=1$ (Kroupa 1992). 


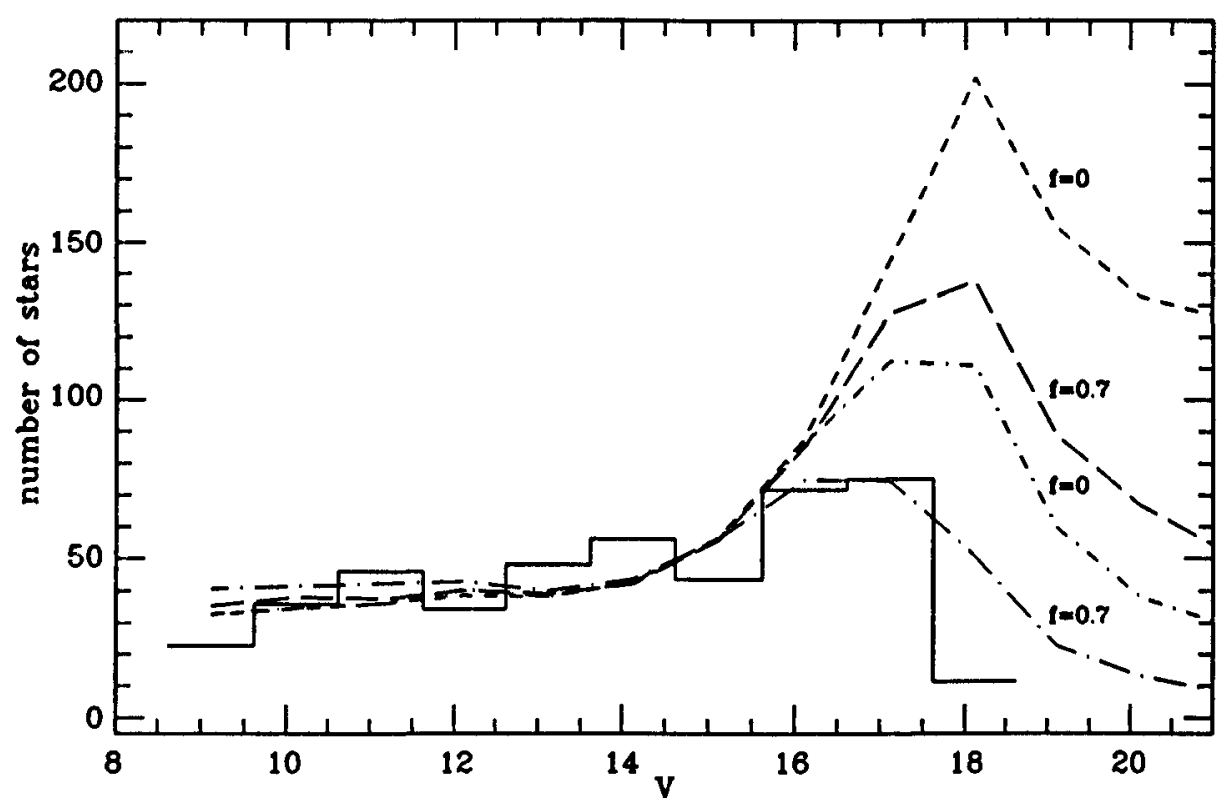

FIGURE 2. Luminosity function for Praesepe: The histogram is the observed data (Jones \& Stauffer 1991) and is incomplete for $V>16$. The dashed lines are for a mass function with $\alpha=1.1$, and the dash-dot-dashed lines for $\alpha=0$.

where $m$ is the stellar mass in solar units, $\alpha \approx 1.2$ and $\xi(m) d m$ is the number of stars per $\mathrm{pc}^{3}$ in the mass range $m$ to $m+d m$. The hydrogen-burning limit is taken to be $0.08 M_{\odot}$. By studying the luminosity function for main-sequence stars and assuming all 'stars' consist of two components we can in principle constrain the mass function for masses smaller than $0.08 M_{\odot}$. The data remain too poor to allow a more precise conclusion than that the best models have $100 \%$ unresolved duplicity with uncorrelated main-sequence stellar component masses chosen from the same mass function. Our Monte-Carlo simulations include a detailed model of the dispersion about the colour-magnitude relation (Kroupa 1992). This dispersion complicates the distance estimation substantially and smears out features in the luminosity function (see also Kroupa, this Colloquium).

Stars in an open cluster lie at the same distance and have the same age and chemical abundance. The effects of binary systems on the stellar luminosity function thus become very apparent at magnitudes fainter than $M_{\mathrm{V}} \approx 13$ (Kroupa et al. 1992, Kroupa 1992), as shown in Figure 2.

\section{CONCLUSIONS}

1. The dip at $M_{\mathrm{V}} \approx 7$ and the peak at $M_{\mathrm{V}} \approx 12$ in the stellar luminosity function can be explained by structure in the mass-luminosity relation.

2. Binary systems have a significant effect on the shape of the faint-star luminosity function. 
3. Comparison of the single-star and system luminosity functions allows the derivation of the mass function and constrains the mass-ratio distribution.

\section{REFERENCES}

Dahn, C.C., Liebert, J., \& Harrington, R.S. 1986, $A J, 91,621$

Jones, B.F. \& Stauffer J. R. 1991, AJ, 102, 1080

Kroupa, P., Tout, C.A., \& Gilmore, G. 1990, MNRAS, 244, 76

Kroupe, P., Tout, C.A., \& Gilmore, G. 1991, MNRAS, 251, 293

Kroupa, P., Gilmore, G., \& Tout, C.A. 1992, AJ, 103, 1602

Kroupa, P. 1992, Ph.D. thesis, Cambridge University

Popper, D.M. 1980, ARA\&A, 18, 115

Rana, N.C. $1987, A 8 A, 184,104$

Reid, N. \& Gilmore, G. 1982, MNRAS, 201, 73

Stobie, R.S., Ishida, K., \& Peacock, J.A. 1989, MNRAS, 238, 709

Wielen, R., Jahreiss, H., \& Krüger, R. 1983, in The Stars and Stellar Luminosity Function, IAU Coll. 76, eds. A.G. Davis Philip and A.R. Upgren (Schenectady: L. Davis Press), p. 163 